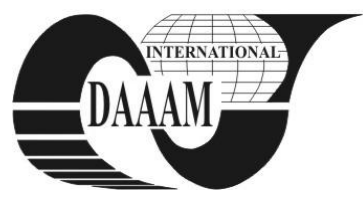

Annals of DAAAM for 2011 \& Proceedings of the 22nd International DAAAM Symposium, Volume 22, No. 1, ISSN 1726-9679 ISBN 978-3-901509-83-4, Editor B. Katalinic, Published by DAAAM International, Vienna, Austria, EU, 2011 Make Harmony between Technology and Nature, and Your Mind will Fly Free as a Bird Annals \& Proceedings of DAAAM International 2011

\title{
FULLWAVE MODELLING OF SHIELDING EFFECTIVENESS OF METALIZED TEXTILES
}

\author{
SKOCIK, P[etr]; GONA, S[tanislav] \& KRESALEK, V[ojtech]
}

\begin{abstract}
In this paper electromagnetic fullwave modelling of metalized textiles is presented. Numerical analysis of the textile having nominal shielding effectiveness $80 \mathrm{~dB}$ is carried out by the commercial fullwave simulation program Ansys based on finite element method. As a result, transmission coeffcient of the metalized textile is obtained from $10 \mathrm{MHz}$ to $10 \mathrm{GHz}$. Results of simulation of shielding effectiveness of the textile show a perfect match with an analytical model at low frequencies. In the frequency range 0.1 to $10 \mathrm{GHz}$ simulated shielding effectiveness is validated by measurements on a TEM coaxial line and also by manufacturer SE data.
\end{abstract}

Key words: metalized textile, shielding effectiveness, fullwave modelling, SE measurements, finite element method

\section{INTRODUCTION}

Several different efficient numerical techniques for calaculation of reflection and transmission from composite materials having circular wires embedded in the epoxy matrix, have been reported in last years (Lin \& Chen., 1993), (Holloway et al., 2005), (Lovat et al., 2008). All these techniques are limited to composite geometries where each plie of the composite has unidirectionally oriented fibers of a circular cross section. In case of geometries having different ply pattern and shape of the cross section of the fiber, fullwave numerical analysis must be used.

Fullwave numerical analysis of the composite materials described in literature is usually limited to cases with a low volume concentration of fibers of circular cross section (Liu et al., 2005). Some authors have reported fullwave modeling of composites having medium concentration of fibers (Volski et al., 2006). However, the original circular wires were replaced by the flat wires, in order to enable use $2.5 \mathrm{MoM}$ codes for prediction of the SE of the composite. This replacement is not possible, for composites having fibers of circular cross section at high volume concentration. Numerical analysis of composites having circular fibers (carbon fiber reinforced composite) at high volume concentration was reported recently (Piche et al., 2009).

In this paper we originally describe new results of fullwave modeling of shielding effectiveness of metalized textiles. These textiles consist from polyamide fibers which are galvanically metalized. Thus, conductive currents on the fiber tend to flow only withing a very thin layer metal area having annulus cross section. In the low frequency range, computed shielding effectiveness shows a perfect match with an analytical model. At higher frequencies simulated results are also compared with measurements on real composite samples.

\section{FEM SIMULATION OF THE METALIZED TEXTILE}

In generall, full wave eletromagnetic analysis of composite materials with respect to its microstructure is a very difficult for two reasons. First, preparation geometrical and finite element model of these structures (on scale of several micrometers or less) is difficult due to a presence of geometrical details (wires, cores, annulus metalization). Second, number of elements that is required to represent geometry is very large. As a result, simulation of these composite structures is time consuming. In order to enable modeling and meshing of small (sub-micron size) structures with extreme aspect ratios, commercial finite element program Ansys was used.

In our research regarding fullwave electromagnetic simulation and measurement of shielding effectiveness of the metalized texlies, several samples supplied by Lorix/Lairdtech company were measured and analyzed by finite element method in Ansys. In this paper, the metalized textile designed as PBN-II 1.3 Cu-Sn is given as an example. The nominal SE of the textile is about $80 \mathrm{~dB}$ at $1 \mathrm{GHz}$ according to the manufacturer data. The textile consists from polyamide microfibers which are galvanically metalized. Fiber thickness was determined with the use of Olympus SX7 microscope. According to a specific picture recognition software the average fiber thickness was measured as 28 micrometers. An average concentration of fibers is about 25 percent. The metalization thickness is extremally small, typically 0.1 micrometers. The example of the physical microstructure of the textile is shown in Fig. 1.

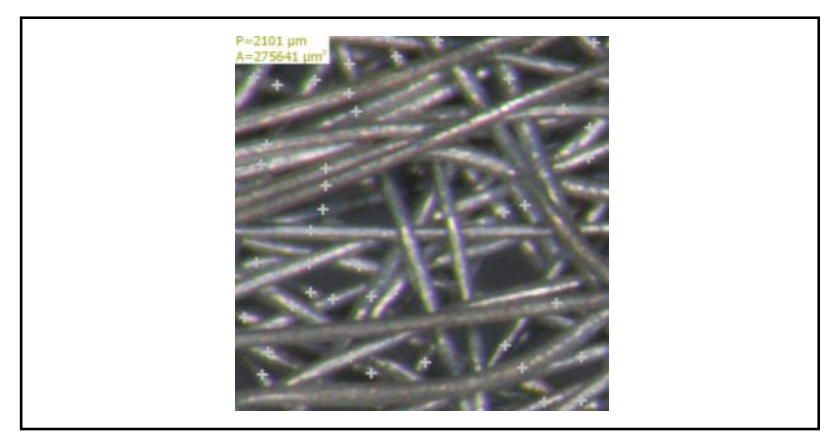

Fig. 1. Metalized textile PBN-II 1.3 Cu-Sn (photograph of microstructure)

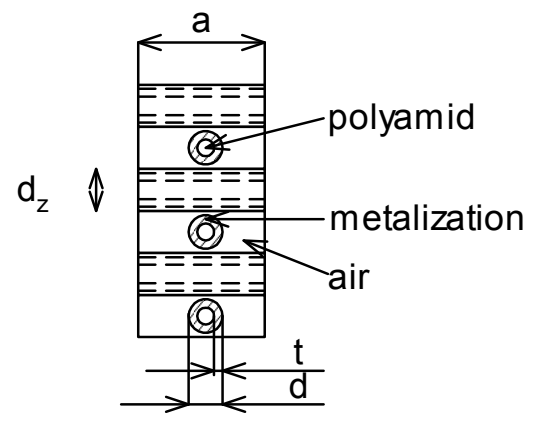

Fig. 2. Periodic model of the metalized textile $(a=75 \mu \mathrm{m}, \mathrm{d}=8$ $\mu \mathrm{m}, \mathrm{t}=0.1 \mu \mathrm{m}, \mathrm{d}_{\mathrm{z}}=33 \mu \mathrm{m}$, conductivity of metallization is assumed $56 \mathrm{MS} / \mathrm{m}$ ) 
The physical structure with arbitrarily oriented fibers (Fig.1) having high volume concentration would be too difficult if not impossible to model in fullwave simulation programs. Thus, the structure of the textile was periodized (Fig. 2). Based on the model from Fig. 2, a corresponding solid and finite element model in Ansys was created (Fig.3 and Fig. 4).

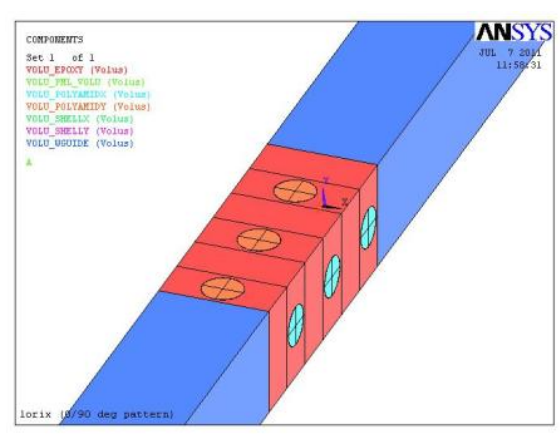

Fig. 3. Solid model of PBN-II Cu-Sn textile in Ansys

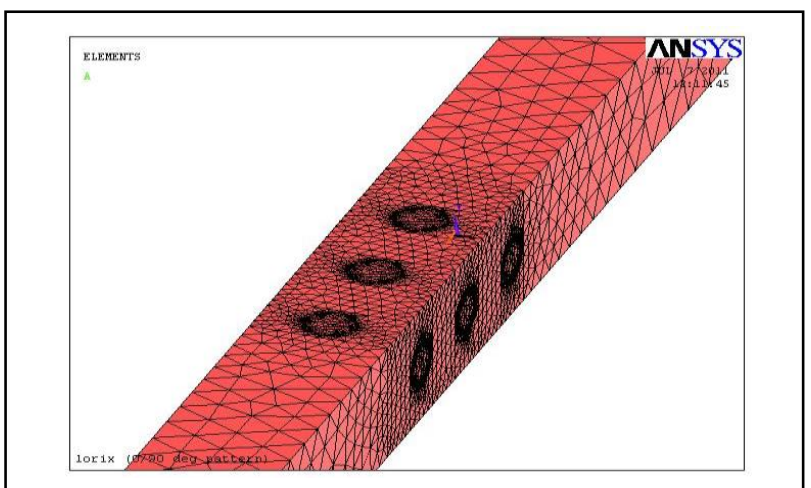

Fig. 4. Finite element model of the PBN-II Cu-Sn textile in Ansys (approx. 500000 tetrahedral $1^{\text {st }}$ order elements)

Shielding effectiveness for the periodic model given in Fig. 2 was simulated in frequency range $10 \mathrm{MHz}$ to $10 \mathrm{GHz}$. At low frequency SE exactly corresponds with analytical low frequency model (Lin \& Chen., 1993). At higher frequencies the SE increases due to the skin effect. Approximately at 5 $\mathrm{GHz}$, a resonance maximum is observed. In the frequency range of several hundreads $\mathrm{MHz}$ a very good agreement between simulated SE and manufacturer SE data is observed.

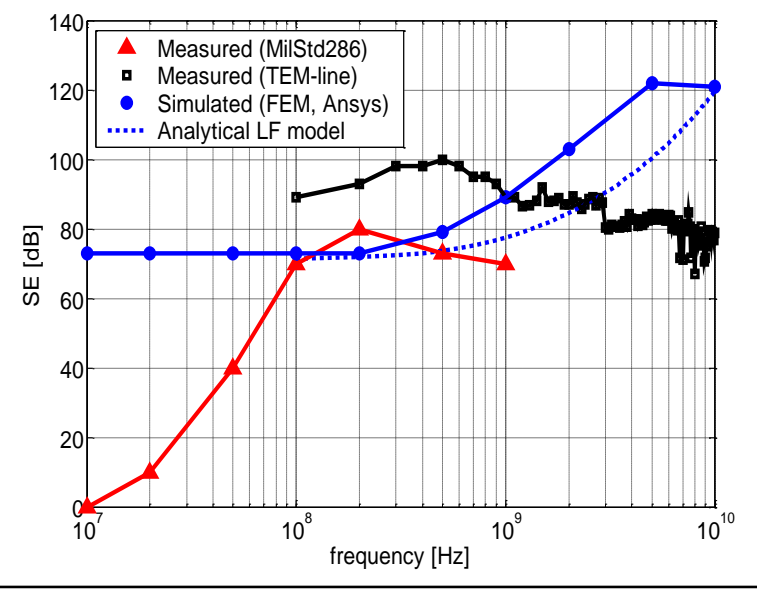

Fig. 5. Comparison between measured and simulated shielding effectiveness of the metalized texstile

The agreement between simulated SE and the shielding effectiveness measured by a coaxial line in frequency range 0.1 to $10 \mathrm{GHz}$ is only approximate. There are 3 principle reasons. First, periodic fibers tend to maximize polarizability of the artificial dielectric / periodic structure. In other words, composites with random fibers do not show so high resonance maximum. Second, the electrogalvanically metallization layer do not have a guranted conductivity at high frequencies, unlike copper cladded material (e.g. copper traces in printed circuit boards) which show conductivity of approx. $60 \mathrm{MS} / \mathrm{m}$ up to several tens of GHz. Third, actual thickness of the metallization might be different from the value given by manufacturer.

\section{CONCLUSION}

Measurement of shielding effectiveness was performed on The TEM coaxial line decribed in (Wieckowski \& Janukiewicz, 2006). The measurement was automated with HP VeePro control program and used the generator SMR20 and the spectrum analyzer FSP40 from Rohde-Schwarz. Results of FEM simulation of shielding effectiveness of metalized textiles were presented and compared with the analytical low frequency model and with the measured SE data.

\section{ACKNOWLEDGEMENTS}

This work was accomplished with financial support by Research Projects of the Ministry of Youth, Education and Sports of the Czech Republic: MSM 7088352102 and by the European Regional Development Fund under the project CEBIA-Tech No. CZ.1.05/2.1.00/03.0089.

\section{REFERENCES}

Holloway, C., L.; Sarto, M.; S. \& Johansson, M. (2008). Analyzing Carbon-Fiber Composite Materials With Equivalent-Layer Models. IEEE Transactions on Electromagnetic Compatibility, Vol. 47, No. 4, (Nov 2005) 833-844, ISSN 0018-9375

Lin, M., S.; Chen, C., H. (1993). Plane-Wave Shielding Characteristics of Anisotropic Laminated. IEEE Transactions on Electromagnetic Compatibility, Vol. 33, No. 1, (Feb 1993) 21-27, ISSN 0018-9375

Liu, L.; Matitsine, S., L.; Gan, Y., B. \& Rozanov, K., N. (2005). Effective permittivity of planar composites with randomly or periodically distributed fibers. Journal of applied physics, Vol. 98, No. 6, (Sep 2005) 80-88, ISSN 0021-8979

Lovat, G.; Burghignoli, P. \& Cellozi, S. (2008). Shielding Properties of a Wire-Medium Screen. IEEE Transactions on Electromagnetic Compatibility, Vol. 50, No. 1, (Feb 2008) 80-88, ISSN 0018-9375

Piche, A.; Bennani, A.; Perraud, R.; Abboud, T.; Béreux, F.; Peres, G.; Srithammavanh \& Srithammavanh, V. (2009). Electromagnetic modeling of multilayer carbon fibers composites, Proceedings of Internation Symposium on Electromagnetic Compatibility - EMC Europe, 11-12 June, Greece, ISBN 978-1-4244-4107-5, Karanasiou, I. (Ed.), pp. 1-4, National Technical University of Athens, Athens

Volski, V.; Aerts, W.; Vasylchenko, O. \& Vandenbosch, G. (2006). Composite Textiles Filled with Arbitrarily Oriented Conducting Fibers using a Periodic Model for Crossed Strips. Proceedings of International Conference on Mathematical Methods in Electromagnetic Theory, 26-29 June, Ukraine, ISBN 1-4244-0490-8, Veliev, E. (Ed.), pp. 58-63, IEEE East Ukraine Joint Chapter, Kharkov

Wieckowski, T., W. \& Janukiewicz, J., M. (2006). Methods for Evaluating the Shielding Effectiveness of Textiles. FIBRES \& TEXTILES in Eastern, Vol. 14, No. 5, (Jan/Feb 2006) 18-22, ISSN 1230-3666 